\title{
Endoscopic Ultrasound-Guided Transgastric Drainage of an Intra- Abdominal Abscess following Gastrectomy
}

\author{
Satoru Kikuchi', Tetsushi Kubota', Shinji Kuroda', Masahiko Nishizaki ${ }^{1}$, Shunsuke Kagawa', Hironari Kato ${ }^{2}$, Hiroyuki Okada ${ }^{2}$, and \\ Toshiyoshi Fujiwara'
}

Department of ${ }^{1}$ Gastroenterological Surgery, ${ }^{2}$ Gastroenterology and Hepatology, Okayama University Graduate School of Medicine, Dentistry and Pharmaceutical Sciences, Okayama, Japan

Endoscopic ultrasound (EUS)-guided transgastric drainage has been performed as a less invasive procedure for pancreatic fistulas and intra-abdominal abscesses occurring after surgery in recent years. However, there are no reports of EUS-guided transgastric drainage of intra-abdominal abscesses following gastrectomy. This case report describes 2 patients who developed an intra-abdominal abscess following gastrectomy and underwent EUS-guided transgastric drainage. Both patients underwent laparoscopy-assisted distal gastrectomy with Billroth-I reconstruction for gastric cancer. The intra-abdominal abscesses were caused by postoperative pancreatic fistula that developed following gastrectomy. One patient underwent naso-cystic drainage and the other underwent only a needle puncture of the abscess cavity. EUS-guided drainage was performed safely and effectively, although 1 patient developed gastroduodenal anastomotic leakage related to this procedure. In summary, EUS-guided transgastric drainage is safe and technically feasible even in post-gastrectomy patients. However, it is necessary to be careful if this procedure is performed in the early period following gastrectomy. Clin Endosc 2019;52:373-376

Key Words: Endoscopic ultrasound; Postoperative intra-abdominal abscess; Transgastric drainage

\section{INTRODUCTION}

A postoperative intra-abdominal abscess associated with a pancreatic fistula and anastomotic leakage is a serious complication of gastrectomy for gastric cancer. ${ }^{1}$ Ultrasonography or computed tomography (CT) guided percutaneous drainage is a less invasive and effective first-line treatment for an intra-abdominal abscess. ${ }^{2}$ However, in a few patients, it is difficult to gain access to the fluid collections via the percutaneous approach because of their location and proximity to

Received: August 6, 2018 Revised: October 8, 2018

Accepted: October 11, 2018

Correspondence: Satoru Kikuchi

Department of Gastroenterological Surgery, Okayama University Graduate School of Medicine, Dentistry, and Pharmaceutical Sciences, 2-5-1 Shikata-cho, Kita-ku, Okayama 700-8558, Japan

Tel: +81-86-235-7257, Fax: +81-86-221-8775, E-mail: satorukc@okayama-u.ac.jp ORCID: https://orcid.org/0000-0002-7671-0696

cc This is an Open Access article distributed under the terms of the Creative Commons Attribution Non-Commercial License (http://creativecommons.org/ licenses/by-nc/3.0) which permits unrestricted non-commercial use, distribution, and reproduction in any medium, provided the original work is properly cited. surrounding visceral organs.

Recently, endoscopic ultrasound (EUS)-guided transmural drainage is a standard procedure performed for pancreatic pseudocysts, ${ }^{3}$ and its applications have been gradually extended to postoperative pancreatic fistulas (POPF) or intra-abdominal abscesses. ${ }^{4,5}$ EUS-guided transmural drainage has been reported in a few patients who present with anatomical alterations following previous gastric surgery. ${ }^{6-8}$ However, no cases of EUS-guided transgastric drainage have been reported for intra-abdominal abscesses following gastrectomy.

\section{CASE REPORTS}

\section{Case 1}

A 29-year-old woman underwent laparoscopy-assisted distal gastrectomy (LADG) with Billroth-I reconstruction for gastric cancer. Although she developed a Grade B POPF based on the International Study Group of Postoperative Pancreatic Fistula, ${ }^{9}$ she improved with conservative therapy and was 
discharged on postoperative day (POD) 11 without any symptoms. However, she was re-admitted on POD 20 with high fever and backache. An encapsulated fluid collection around the remnant stomach was detected on abdominal contrast-enhanced (CE) CT (Fig. 1A). The fluid collection was diagnosed as an intra-abdominal abscess associated with POPF. Her condition did not improve with antibiotic therapy; therefore, EUS-guided transgastric drainage was performed on POD 22 because a percutaneous approach was difficult without injuring the surrounding visceral organs. The EUS-guided procedure was performed using a convex array echoendoscope (GF-UCT260; Olympus Medical Systems, Tokyo, Japan). The abscess cavity identified by the EUS (Fig. 1B) was smaller than that identified by CT because of spontaneous perforation into the gastric lumen (Fig. 1C), and only a puncture of the abscess cavity was performed using a 19-gauge needle (Echo Tip; Cook Medical, Tokyo, Japan) without placement of a drainage catheter. The abscess cavity collapsed after the aspiration of a small amount of white viscous purulent fluid. The patient's symptoms improved immediately after drainage, and she was discharged 2 weeks after drainage. CT performed a month after EUS-guided drainage did not reveal any fluid collections (Fig. 1D).

\section{Case 2}

A 73-year-old man underwent LADG with Billroth-I reconstruction for gastric cancer and partial colectomy for transverse colon cancer. He developed a high fever and abdominal pain on POD 6, and CT showed swelling of the body of the pancreas and fluid collection around the remnant stomach (Fig. 2A, B). This fluid collection was diagnosed as POPF related to lymph node dissection performed for gastric cancer, and the patient's condition did not improve with antibiotic and protease inhibitor treatment. Thus, EUS-guided transgastric drainage was performed. The EUS-guided procedure was performed using a convex array echoendoscope (GF-UCT260). EUS showed a large monolocular cyst $(50 \times 30$ $\mathrm{mm})$ at the dorsal aspect of the remnant stomach. Following puncture of the cyst using a 19-gauge needle (Echo Tip), 15 $\mathrm{mL}$ of white viscous purulent fluid was aspirated. Successful
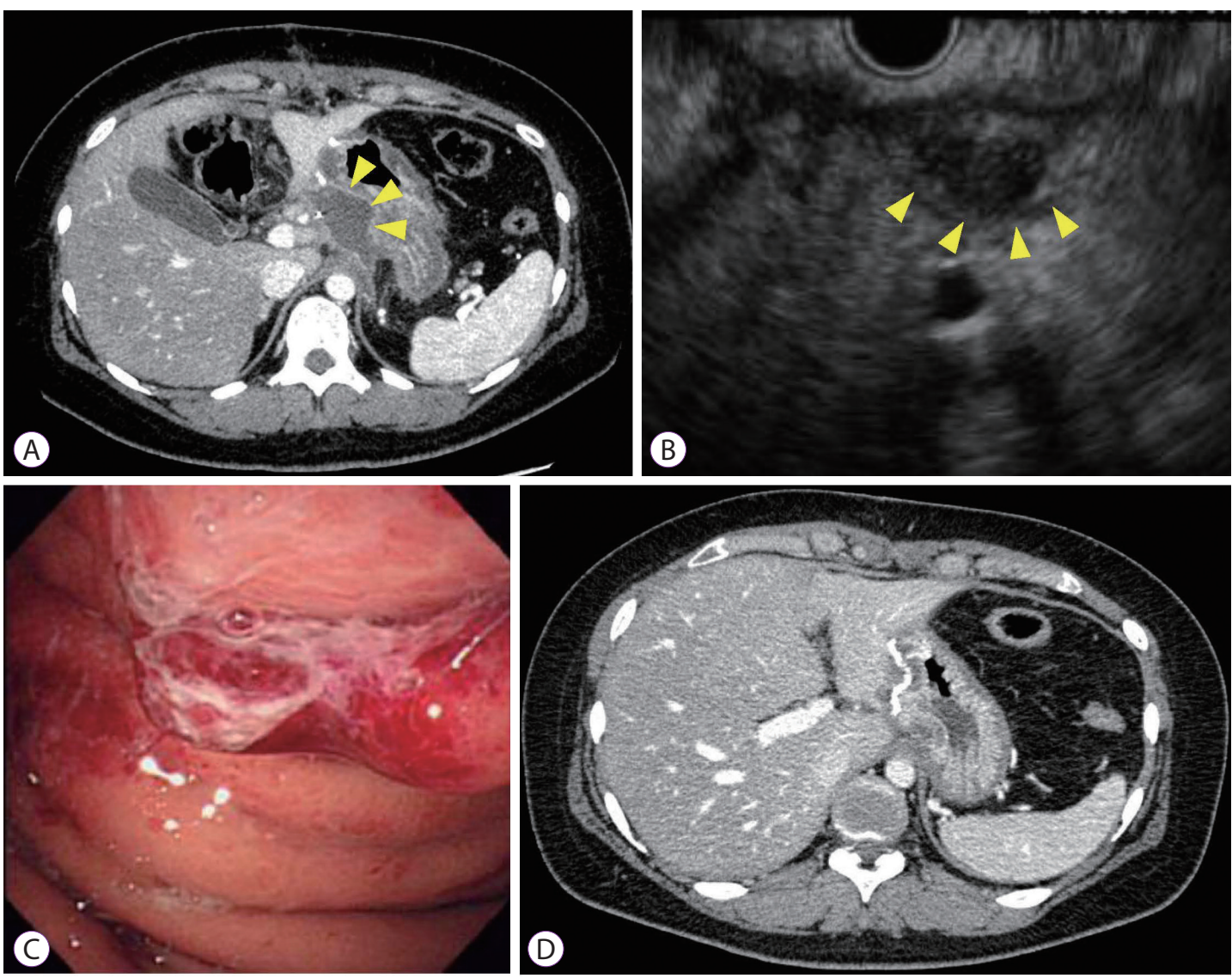

Fig. 1. Imaging findings in Case 1 show the following features: (A) Computed tomography (CT) scan shows an intra-abdominal abscess around the remnant stomach (arrowheads). (B) Endoscopic ultrasound (EUS) image shows a small cloudy fluid collection around the stomach (arrowheads). (C) Endoscopic examination shows red, bulging mucosa on the posterior wall of the remnant stomach. (D) One month after the EUS-guided drainage, the CT scan shows no fluid collection around the stomach. 
access to the abscess was confirmed by injection of contrast agent, and a 0.035-inch guidewire (Jagwire; Boston Scientific, Tokyo, Japan) was introduced through the needle into the abscess cavity. Subsequently, the fistula was dilated using a 7-Fr dilation catheter (Soehendra Biliary Dilation Catheter; Cook Medical), and a 7-Fr pigtail nasal biliary catheter (Cook Medical) was deployed into the abscess cavity on POD 8 (Fig. 2C, D). The patient's condition improved promptly, and the abscess cavity decreased in size after drainage; however, gastroduodenal anastomotic leakage was observed immediately after EUS-guided transgastric drainage. Subsequently, the patient developed bleeding from a pseudoaneurysm of the common hepatic artery 10 days after drainage, which was successfully treated with coil embolization performed by an interventional radiologist (Fig. 2E). The nasal catheter was removed 13 days after drainage, and the patient was discharged on POD 47. During the 2-year follow-up period, the patient showed no evidence of abscess recurrence.

\section{DISCUSSION}

This report describes EUS-guided transgastric drainage of intra-abdominal abscesses following gastrectomy. Technical and clinical success was achieved in both patients. The clinical success rates of percutaneous and EUS-guided drainage of intra-abdominal abscesses were reported to be $65 \%-90 \%$ and $80 \%$, respectively. ${ }^{10,11}$ It has been reported that the time required to achieve clinical success with EUS-guided transgastric drainage was significantly shorter than that with percutaneous drainage. ${ }^{5}$ In the present cases, we selected EUS-guided transgastric drainage because a percutaneous approach to the abscesses was difficult without surrounding visceral organ injury, and the abscess cavity and the remnant stomach wall were in close contact.

Although no complications related to this procedure, including bleeding, perforation, and visceral organ injury, were reported, gastroduodenal anastomotic leakage was observed immediately following the procedure in Case 2. Determining the appropriate time for drainage is important because transmural drainage of the fluid collection without a mature abscess wall may cause intraperitoneal spillage. A few studies have reported EUS-guided drainage of fluid collections within 4 weeks postoperatively. ${ }^{5,12}$ However, Tilara et al. ${ }^{4}$ reported that early EUS-guided drainage of abdominal fluid collections within 30 days after pancreatic resection was safe and not
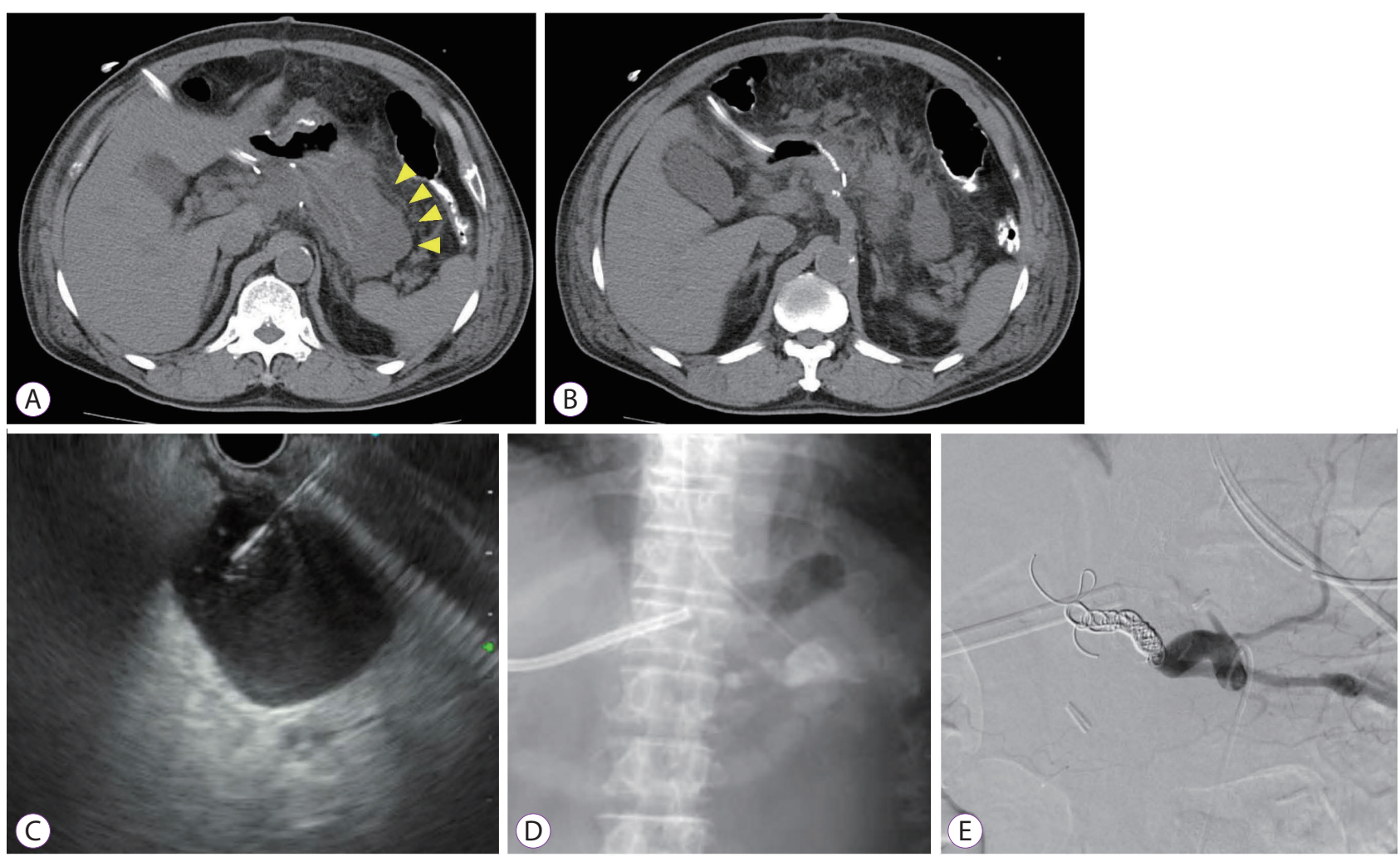

Fig. 2. Imaging findings in Case 2 show the following features: (A, B) Computed tomography scan shows a swelling of the body of the pancreas and a fluid collection around the remnant stomach (arrowheads). (C) Endoscopic ultrasound image shows a huge unilocular cyst close to the remnant stomach, which was punctured using a needle. (D) A fluoroscopic image shows the placement of a 7-Fr pigtail nasal biliary catheter. (E) An angiographic image shows that the bleeding from a pseudoaneurysm of the common hepatic artery has been successfully treated with coil embolization. 
associated with increased complications. Gupta et al. reported that the median time from the diagnosis of the fluid collections to transmural drainage was 4 days. ${ }^{11}$ In the present case series, we confirmed a fluid collection with a mature abscess wall that showed enhancement on CE-CT before drainage. Moreover, the abscess cavity wall was confirmed by EUS before drainage. It is important to confirm whether the capsule is formed around the abscess before transmural drainage is performed. Although we could not accurately confirm the cause of the anastomotic leakage after drainage, the anastomotic site was located close to the drainage site because of the gastrectomy, and the anastomotic site might have been stretched during the drainage procedure. Additionally, prolonged exposure to pancreatic juice might have damaged the anastomotic site. Moreover, in the patient with complications, EUS-guided drainage was performed 8 days after gastrectomy, which may be too early to perform this procedure. Notably, EUS-guided drainage (a less invasive procedure), was selected because the option of waiting for the intra-abdominal abscess to fully organize was not available and only EUS-guided drainage or surgical drainage could be performed. There is no ideal time for drainage, including for EUS-guided drainage, and the optimal time depends on the patient's clinical condition.

A naso-cystic drainage tube or an internal plastic stent is usually placed in patients undergoing EUS-guided drainage of infectious cysts. ${ }^{3}$ In the present cases, a 7-Fr pigtail naso-cystic drainage tube was placed in Case 2. However, in Case 1, only a puncture of the abscess was performed without placement of a drainage tube because the abscess cavity was small $(16 \mathrm{~mm})$ and it was expected to resolve immediately after drainage. The ideal drainage method is dependent on the location and size of the individual abscess cavity. However, a naso-cystic drainage tube is more useful for lavage of the cavity when the fluid collection is infected. In Case 2, a naso-cystic drainage tube was selected because the fluid collection was diagnosed as an infected abscess when the puncture was performed, and the naso-cystic drainage tube was used for lavage a few times after drainage.

In conclusion, this case report suggests that EUS-guided transgastric drainage of intra-abdominal abscesses is safe and technically feasible even in post-gastrectomy patients. However, it is necessary to be careful when performing this procedure in the early period following gastrectomy.
Conflicts of Interest

The authors have no financial conflicts of interest.

\section{Acknowledgements}

This work was supported by the Japan Society for the Promotion of Science Grant-in-Aid for Young Scientists, Grant Number 18K16362.

\section{Author Contributions}

Conceptualization: Satoru Kikuchi, Tetsushi Kubota

Data curation: SKi, TK, Shinji Kuroda, Hironari Kato

Formal analysis: Masahiko Nishizaki, Shunsuke Kagawa, Hiroyuki Okada, Toshiyoshi Fujiwara

Writing-original draft: SKi

Writing-review\&editing: TK, SKu, MN, HK, SKa, HO, TF

\section{REFERENCES}

1. Sasako M, Sano T, Yamamoto S, et al. D2 lymphadenectomy alone or with para-aortic nodal dissection for gastric cancer. N Engl J Med 2008;359:453-462.

2. Kassi F, Dohan A, Soyer P, et al. Predictive factors for failure of percutaneous drainage of postoperative abscess after abdominal surgery. Am J Surg 2014;207:915-921.

3. Itoi T, Itokawa F, Tsuchiya T, Kawai T, Moriyasu F. EUS-guided pancreatic pseudocyst drainage: simultaneous placement of stents and nasocystic catheter using double-guidewire technique. Dig Endosc 2009;21 Suppl 1:S53-S56.

4. Tilara A, Gerdes H, Allen P, et al. Endoscopic ultrasound-guided transmural drainage of postoperative pancreatic collections. J Am Coll Surg 2014;218:33-40

5. Onodera M, Kawakami H, Kuwatani M, et al. Endoscopic ultrasound-guided transmural drainage for pancreatic fistula or pancreatic duct dilation after pancreatic surgery. Surg Endosc 2012;26:1710-1717.

6. Itoi T, Itokawa F, Sofuni A, Umeda J, Tsuchiya T. Late bleeding after EUS-guided transjejunal drainage of a pancreatic pseudocyst in a Rouxen-Y patient. Dig Endosc 2011;23 Suppl 1:51-53.

7. Larghi A, Seerden TC, Galasso D, et al. EUS-guided cystojejunostomy for drainage of a pseudocyst in a patient with Billroth II gastrectomy. Gastrointest Endosc 2011;73:169-171.

8. Varadarajulu S, Tamhane A, Blakely J. Graded dilation technique for EUS-guided drainage of peripancreatic fluid collections: an assessment of outcomes and complications and technical proficiency (with video). Gastrointest Endosc 2008;68:656-666.

9. Bassi C, Dervenis C, Butturini G, et al. Postoperative pancreatic fistula: an international study group (ISGPF) definition. Surgery 2005;138:8-13.

10. van Sonnenberg E, Wittich GR, Goodacre BW, Casola G, D’Agostino HB. Percutaneous abscess drainage: update. World J Surg 2001;25:362369; discussion 370-372.

11. Gupta T, Lemmers A, Tan D, Ibrahim M, Le Moine O, Deviere J. EUS-guided transmural drainage of postoperative collections. Gastrointest Endosc 2012;76:1259-1265.

12. Varadarajulu S, Wilcox CM, Christein JD. EUS-guided therapy for management of peripancreatic fluid collections after distal pancreatectomy in 20 consecutive patients. Gastrointest Endosc 2011;74:418-423. 